\title{
In Situ TEM Studies of Nanoparticle Self-Assembly: Imaging the Evolution of Pulsed-Laser-Induced Dewetting Processes
}

\author{
Joseph T. McKeown ${ }^{1}$, Yueying $\mathrm{Wu}^{2}$, Jason D. Fowlkes ${ }^{3}$, Thomas LaGrange ${ }^{1}$, Bryan W. Reed ${ }^{1}$, Philip \\ D. Rack $^{2,3}$, and Geoffrey H. Campbell ${ }^{1}$ \\ ${ }^{1}$ Condensed Matter and Materials Division, Lawrence Livermore National Laboratory, Livermore, CA, \\ USA \\ ${ }^{2}$ Department of Materials Science and Engineering, University of Tennessee, Knoxville, TN, USA \\ ${ }^{3}$ Center for Nanophase Materials Science, Oak Ridge National Laboratory, Oak Ridge, TN, USA
}

The synthesis and organization of functional nanostructures using self-assembly processes embodies a critical challenge for nano- and meso-science, as two- and three-dimensional (2D and 3D) spontaneous arrangement of nanoscale structural units or building blocks can produce ordered ensembles that exhibit collective functionality at reduced cost with greater efficiency [1,2]. Generating arrays of nanoparticles with 2D planar and 3D configurations is key to this challenge, and self-assembly techniques can play a key role if they can be directed to express specific patterned structures reliably and reproducibly.

The ability to monitor and characterize self-assembly processes in situ while the system is evolving can potentially lead to increased control and directed self-assembly. However, in situ characterization of the dynamics of self-assembly processes has proven extremely challenging, particularly in materials systems that utilize metallic nanoparticles, as the dynamics of these processes evolve extremely fast over very small spatial scales, the combination of which are beyond the capabilities of conventional characterization techniques. Here, we present results from in situ characterization experiments that reveal the assembly dynamics during pulsed-laser-induced dewetting of metallic thin films using the high spatiotemporal resolution of the dynamic transmission electron microscope (DTEM) [3] at Lawrence Livermore National Laboratory.

Figure 1 presents time-resolved, 20-ns exposure DTEM images of a $\mathrm{Cu}-\mathrm{Co}$ film as it dewets a silicon nitride substrate upon pulsed-laser melting. The time-delay sequence of images shows progressive hole formation in the liquid film and subsequent break-up, leading to an array of self-assembled nanoparticles. Results from both $\mathrm{Cu}-\mathrm{Co}$ and pure $\mathrm{Ni}$ thin films will be presented. The self-assembly mechanisms in both materials systems will be discussed, and implications for directing nanoparticle assembly in pure metallic and alloy thin films will be examined [4].

\section{References:}

[1] M. Antonietti, G.A. Ozin, Chemistry A European Journal 10 (2004) 28.

[2] G.M. Whitesides et al., Self-Assembly and Nanostructured Materials, in Nanoscale Assembly:

Chemical Techniques, W.T.S. Huck, Ed. (Springer: Neew York, 2005).

[3] McKeown, J.T. et al., Langmuir 28 (2012) 17168.

[4] Work at Lawrence Livermore National Laboratory was performed under the auspices of the U.S. Department of Energy, Office of Science, Office of Basic Energy Sciences, Division of Materials Sciences and Engineering under Contract DE-AC52-07NA27344. Work at the University of Tennessee was supported by the U.S. Department of Energy, Office of Science, Office of Basic Energy Sciences, Division of Materials Sciences and Engineering. Research conducted at the Center for Nanophase 
Materials Sciences is sponsored at Oak Ridge National Laboratory by the Scientific User Facilities Division of the U.S. Department of Energy, Office of Science, Office of Basic Energy Science.

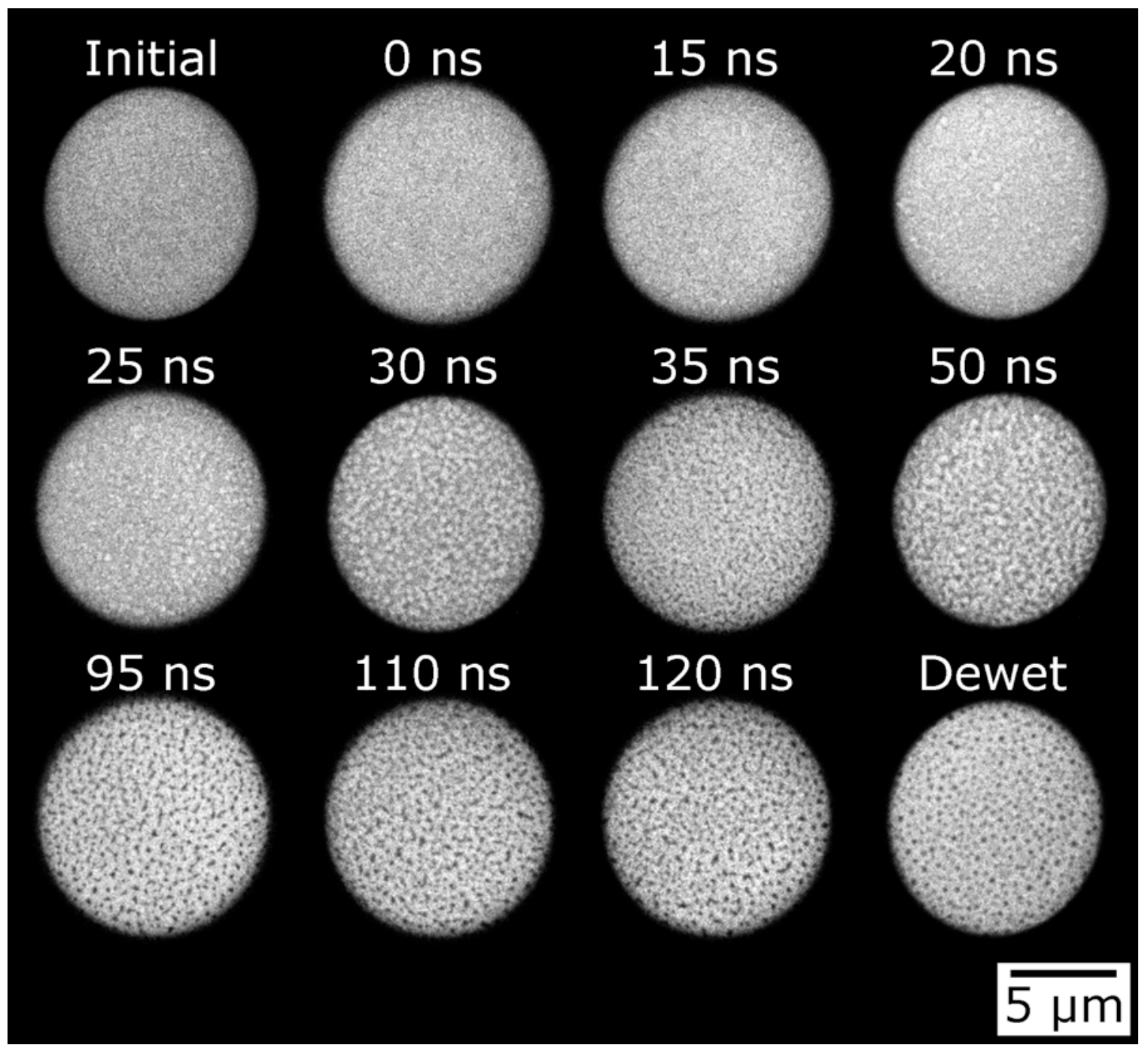

Figure 1. Dynamic time-delay sequence of images recorded as a 10-nm-thick, equiatomic Cu-Co thin film dewets a silicon nitride membrane upon pulsed-laser melting. The indicated times above each image are the delays between the peak of the Gaussian laser pulse that melted the film and the 20-ns electron pulse used to form the image. 